\title{
Parenteral and enteral feeding in preterm piglets differently affects extracellular matrix proteins, enterocyte proliferation and apoptosis in the small intestine
}

\author{
Marijke Oste ${ }^{1}$, Maartje De Vos ${ }^{1}$, Els Van Haver ${ }^{1}$, Leen Van Brantegem ${ }^{1}$, Thomas Thymann ${ }^{2}$, Per Sangild ${ }^{2}$, \\ Andre Weyns ${ }^{1}$ and Chris Van Ginneken ${ }^{1 *}$ \\ ${ }^{1}$ Laboratory of Veterinary Anatomy and Embryology, Department of Veterinary Medicine, University of Antwerp, 2610 Wilrijk, \\ Belgium \\ ${ }^{2}$ Department of Human Nutrition, Faculty of Life Sciences, University of Copenhagen, DK-1958 Frederiksberg C, Denmark
}

(Received 10 December 2009 - Revised 17 March 2010 - Accepted 24 March 2010 - First published online 19 May 2010)

\begin{abstract}
The preterm intestine is immature and responds differently to total parenteral nutrition (TPN) and enteral nutrition, compared with the term intestine. We hypothesised that in preterms, diet composition and feeding route affect mucosal morphology, enterocyte mitosis and apoptosis, and the distribution of laminin-1, fibronectin and collagen IV (extracellular matrix proteins (ECMP)). Preterm piglets (93.5\% of gestation) were delivered via caesarean section and birth weight-matched allocated to one of the four experimental groups: the piglets were either euthanised immediately after delivery, after $3 \mathrm{~d}$ of TPN or after $2 \mathrm{~d}$ enteral feeding with colostrum or milk formula, following $3 \mathrm{~d}$ of TPN. We combined immunohistochemistry, image analysis and stereological measurements to describe the intestinal mucosal layer. No significant changes occurred after $3 \mathrm{~d}$ of TPN. Feeding colostrum or milk replacer for $2 \mathrm{~d}$ after TPN was associated with an increased crypt depth. Only enteral feeding with colostrum resulted in an increased villus height and mitotic index. Neither TPN nor enteral feeding changed the distribution pattern of ECMP or the occurrence of bifid crypts. The immature distribution pattern of ECMP in TPN-fed piglets, coupled with unchanged enterocyte mitosis and apoptosis indices, illustrates that feeding preterm pigs $3 \mathrm{~d}$ TPN does not lead to mucosal atrophy. Despite the invariable distribution of ECMP, colostrum was associated with crypt hyperplasia resulting in an increased villus height. These data illustrate that some mechanisms regulating cell turnover are immature in preterms and may in part explain the abnormal gut responses to TPN and enteral feeding in prematurely born pigs.
\end{abstract}

Preterm piglets: Crypt fission: Extracellular matrix proteins: Cell turnover

The newborn's digestive tract faces the challenge of an abrupt transition from intra-uterine parenteral nutrition support via the umbilical cord to extra-uterine enteral nutrition with mother's milk or formula. In order to meet this goal, the gastrointestinal tract undergoes intensive growth several weeks before delivery ${ }^{(1)}$. In consequence, preterm neonates have an immature gastrointestinal tract and often require a period of total parenteral nutrition (TPN) - in combination with minimal enteral feeding - before total enteral nutrition can be administered $^{(2)}$. Fortunately, it was shown in a study using the preterm piglet as a model of human preterm nutrition that the preterm intestine is less sensitive to TPN-induced mucosal atrophy compared with the term gut ${ }^{(1,3,4)}$. A study conducted by Niinikoski et al. attributed the TPN-induced mucosal atrophy seen in term-born neonates to a dramatic decrease in intestinal blood flow lowering protein synthesis, cell survival and proliferation ${ }^{(5)}$. This detrimental effect of TPN was already visible after $48 \mathrm{~h}$ of TPN.

The prenatal growth of the mucosa continues after birth in term and preterm piglets, especially after the administration of colostrum $^{(1,3-8)}$. In term piglets, milk formula is well tolerated and induces normal intestinal growth and villus elongation without the development of intestinal atrophy and inflammation ${ }^{(6,7)}$. In contrast, in preterm piglets, feeding formula induces mucosal atrophy and intestinal dysfunction both with and without a previous TPN period ${ }^{(1,3,4)}$. In contrast to the situation in the term piglet, the mechanisms - enterocyte proliferation, enterocyte apoptosis, crypt fission, extracellular matrix proteins (ECMP) - supporting these changes in mucosal morphology and their relation to diet or feeding route are not yet documented in detail in the preterm piglet.

Mucosal surface growth is coupled to the enterocyte proliferation rate. Wolinski et al. observed a transient elevation of the mitosis:apoptosis ratio in jejunal enterocytes, resulting in growth of the gut mucosa after enteral feeding for $6 \mathrm{~d}$ after delivery ${ }^{(8)}$. In addition to proliferation, crypt fission could add to this increase of the epithelial surface ${ }^{(9,10)}$. Already it has been shown that dietary luminal factors influence the proliferation and apoptosis rate of enterocytes ${ }^{(8,11-14)}$ and - albeit not always in the same manner - the process of

Abbreviations: COL, oral nutrition with sow's colostrum; ECMP, extracellular matrix proteins; FOR, oral nutrition with an infant milk formula; TPN, total parenteral nutrition; UFD, no nutrition; $\mathrm{V}_{\mathrm{vapop}}$, volume density of apoptotic cells; $\mathrm{V}_{\mathrm{vprol}}$, volume density of mitotic (proliferative) cells.

* Corresponding author: Professor Dr C. Van Ginneken, fax +32 326524 33, email chris.vanginneken@ua.ac.be 
crypt fission ${ }^{(9,14-16)}$. In addition, interactions between the enterocytes and components of the extracellular matrix affect epithelial cell mitosis ${ }^{(17-20)}$. The role of the mesenchymal environment in the process of regulating epithelial cell proliferation via crypt fission seems minor ${ }^{(20)}$. ECMP form an organised network of glycoproteins, proteoglycans and glycosaminoglycans of which type IV collagen, laminins and fibronectin are the main representatives and constituents of the basement membrane ${ }^{(21)}$. A balanced distribution of ECMP steers the differentiation and migration of enterocytes along the crypt-villus axis ${ }^{(17-20)}$. However, their composition and distribution can be altered during gastrointestinal disease processes, resulting in delayed mucosal healing and thus lesion formation ${ }^{(22,23)}$.

Considering the importance of an adequate cell renewal cycle for gut mucosal growth and repair in preterm newborns, we aimed to investigate the underlying processes: mitosis and apoptosis of the enterocytes, crypt fission and the concurrent alteration in the ECMP in preterm piglets subjected to TPN immediately after delivery, followed by enteral nutrition. We used image analysis techniques and morphometry based upon stereological principles in order to generate unbiased quantitative data.

We hypothesise that the relative insensitivity of preterm pigs for mucosal atrophy is due to a low proliferation and apoptotic capacity of immature enterocytes and a non-gradient distribution of the ECMP along the villus axis. Introduction of enteral feeding might affect these processes depending on the nature of the enteral feed (formula $v$. sow's colostrum).

\section{Experimental methods}

\section{Animals}

Delivery, incubation, surgery and assignment to feeding patterns of the premature piglets were implemented according to methods described earlier ${ }^{(3,4)}$. In short, a total of twentynine crossbred (Large White $\times$ Danish Landrace) piglets from six sows were delivered by caesarean section at 107$108 \mathrm{~d}(93-94 \%)$ of gestation. After delivery, the piglets were placed in infant incubators with regulated temperature, moisture and extra $\mathrm{O}_{2}$ supply. Throughout the experiment rectal temperature and $\mathrm{O}_{2}$ saturation were monitored. In contrast to man, transplacental transport of immunoglobulins does not occur in pigs, which makes neonatal pigs dependent on colostral immunoglobulins for passive immunisation. Therefore all pigs were passively immunised with maternal plasma $(4 \mathrm{ml} / \mathrm{kg}$ at $8 \mathrm{~h}, 5 \mathrm{ml} / \mathrm{kg}$ at $16 \mathrm{~h}$, and $10 \mathrm{ml} / \mathrm{kg}$ at $24 \mathrm{~h}$ post-partum) infused via the umbilical catheter. Maternal blood was collected from a uterine vein immediately after the caesarean section and plasma was separated aseptically after centrifugation $\left(4000 \mathrm{~g} ; 4^{\circ} \mathrm{C} ; 10 \mathrm{~min}\right)$. This method yielded plasma levels of immunoglobulins as reported previously $^{(1,3)}$ and was shown sufficient to exclude any systemic infections over the experimental period.

The piglets were randomly assigned to experiment 1 or 2 . The National Committee on Animal Experimentation, Denmark, and the Ethical Committee on Animal Experimentation of the University of Antwerp, Belgium, approved these experiments and the institutional and national guidelines for the care and use of animals were followed.
Experiment 1. After delivery, the piglets were birth weight-matched assigned to two different groups. One group of piglets was killed within $12 \mathrm{~h}$ of delivery and received no nutrition (UFD; $n 3$ ). A second group of piglets was given an elemental TPN ( $n$ 6) fluid (Nutriflex Lipid plus; Braun, Melsungen, Germany) for $3 \mathrm{~d}$. The parenteral nutrient solution was prepared aseptically (Nutriflex Lipid plus; Braun). The composition was based on that used previously for term and preterm pigs $^{(3,4)}$. In order to achieve this composition, part of the glucose and lipid was withdrawn and substituted with amino acids (Vamin $18 \mathrm{~g}$ N/litre electrolyte free; Fresenius Kabi, Uppsala, Sweden) and sterile water. The nutrient composition and concentrations of macrominerals per litre were as follows: total energy, $3123 \mathrm{~kJ}$; glucose, $72 \mathrm{~g}$; lipids, $31 \mathrm{~g}$; $\mathrm{N}, 6.4 \mathrm{~g}$, amino acids, $45 \mathrm{~g}$; Na, $0.88 \mathrm{~g} ; \mathrm{K}, 1.03 \mathrm{~g}$; Ca, $0.13 \mathrm{~g}$; $\mathrm{P}, 0 \cdot 37 \mathrm{~g}$. The TPN solution was infused continuously via a catheter inserted into the umbilical vein (TPN group) using automatic infusion pumps (Infusomat Secura, Braun). The nutritional goal was to provide the pigs with sufficient energy and protein to allow for a slightly positive energy balance. The infusion rate was $4 \mathrm{ml} / \mathrm{kg}$ per $\mathrm{h}$ for the first $24 \mathrm{~h}$ post-partum, gradually increasing to $6-8 \mathrm{ml} / \mathrm{kg}$ per $\mathrm{h}$ during the subsequent $2 \mathrm{~d}$. In this way, the pigs received an average of $450 \mathrm{~kJ} / \mathrm{kg}$ per $\mathrm{d}, 6.5 \mathrm{~g}$ amino acids $/ \mathrm{kg}$ per $\mathrm{d}$ and a fluid intake of $160 \mathrm{ml} / \mathrm{kg}$ per d during the $3 \mathrm{~d}$ period.

Experiment 2. After TPN (similar to the groups in experiment 1), piglets were given oral nutrition $(15 \mathrm{ml} / \mathrm{kg}$ per $3 \mathrm{~h}$ ) with either sow's colostrum (COL; $n$ 9) or an infant milk formula (FOR; $n$ 11) for $32-42 \mathrm{~h}$. The milk diets were collected and prepared as described previously ${ }^{(3,4)}$.

\section{Tissue preparation}

The small intestine from the pyloric sphincter to the ileocaecal junction was excised by cutting along the mesenteric border. It was carefully emptied of its contents, placed on an ice-cold metal plate and divided into three equally long segments, designated proximal, middle and distal small intestine. The three segments were weighed and, from the middle of each segment, tissue samples of $2-3 \mathrm{~cm}$ were taken. These were rinsed in 0.01 M-PBS solution, $\mathrm{pH} 7.4$ (PBS) and fixed in $4 \%$ freshly prepared paraformaldehyde $(0 \cdot 1 \mathrm{M}), \mathrm{pH} 7 \cdot 4$. Afterwards the fixative was washed out with PBS over $24 \mathrm{~h}$ and the samples were stored in PBS containing $0.01 \%$ $\mathrm{NaN}_{3}$ at $4^{\circ} \mathrm{C}$.

At random positions, one (3-5 $\mathrm{mm}$ diameter) biopsy was taken from each small-intestinal segment and routinely processed for paraffin embedding. From these tissue blocks, $5 \mu \mathrm{m}$ paraffin vertical sections were made to be either conventionally (haematoxylin-eosin) or immunohistochemically stained after dewaxing and rehydrating with distilled water or PBS, respectively.

\section{Histological scoring and basic morphometry}

The paraffin sections stained with haematoxylin-eosin were used for microscopic scoring of the gastrointestinal lesions according to the presence of atrophic or inflammatory morphological lesions ${ }^{(24)}$ : 0 , normal morphology; 1 , slight tunica mucosa and lamina propria separation; 2, increased tela submucosa and lamina propria separation with oedema in the 
tela submucosa; 3 , severe separation of the tela submucosa and lamina propria, blood congestion and regional loss of villi; 4, blood congestion, transmural necrosis and generalised loss of villi (Fig. 1). In addition, these sections were used for measuring the depth of the crypts and the height of the intestinal villi in thirty well-orientated villi and crypts (per tissue block) at a low magnification $(10 \times)$ and the percentage of crypt fission at a higher magnification $(20 \times)$. Crypt fission is expressed as the percentage of crypts having a split or fissure in their base. Of each tissue block, approximately 100 crypts were scored in a total of thirty sections taken systematically at random. Only crypts sectioned longitudinally were evaluated.

\section{Immunohistochemistry}

Immunohistochemistry (Table 1) was used to detect the ECMP: laminin-1, fibronectin, collagen IV, apoptotic (cleaved caspase-3 protein) and mitotic (proliferating cell nuclear antigen) cells. After antigen retrieval or biotin blocking, non-specific staining was blocked successively by incubation in $3 \% \mathrm{H}_{2} \mathrm{O}_{2}$ and $20 \%$ normal serum. All immunohistochemical incubations were carried out in a closed humidified container. All immunohistochemical steps were alternated by washings in $0.01 \%$ 2-amino-2-hydroxymethyl-propane1,3-diol (Tris)-buffered saline (TBS; $\mathrm{pH} 7 \cdot 4)$. All antisera were diluted in $0.01 \%$ TBS. A positive immunohistochemical reaction was visualised by incubating the sections with 3-amino-9-ethylcarbazole (DakoCytomation, Glostrup, Denmark) for $5 \mathrm{~min}$. Finally, the sections were counterstained with haematoxylin.

\section{Stereological measurements and image analysis}

An Olympus BX50 microscope, equipped with a Sony CCD camera connected to a computer system running the software program Cast-grid (Olympus, Copenhagen, Denmark), was used for the quantitative analyses. The optimal density of the stereological grid (number of points), the number of sections and the number of sample fields were estimated as described previously ${ }^{(25)}$ and resulted in the analysis of approximately thirty fields of vision in thirty sections taken systematically at random of each tissue block.

The volume densities $\left(\mathrm{V}_{\mathrm{v}}\right)$ of the apoptotic cells $\left(\mathrm{V}_{\mathrm{vapop}}\right)$ and mitotic (proliferative) cells $\left(\mathrm{V}_{\mathrm{vprol}}\right)$ in the mucosal layer of the intestinal wall were estimated by counting the number of grid points $(\mathrm{P})$ hitting the space of interest $(\mathrm{P}(\mathrm{I})$ ) (immunoreactive cells lying within or intersecting the margins of the mucosal epithelium of the intestinal wall) and the number of points hitting the reference space $(\mathrm{P}(\mathrm{ref})$ ) (the enterocytes of the mucosal layer). The following equation yielded the $\mathrm{V}_{\text {vapop }}$ and $\mathrm{V}_{\text {vprol }}$ : $\mathrm{Vv}(\mathrm{I} ;$ ref $)=\mathrm{P}(\mathrm{I}) / \mathrm{P}($ ref $)$.

The measurements of the $\mathrm{V}_{\text {vapop }}$ and $\mathrm{V}_{\mathrm{vprol}}$ are comparable with the apoptotic and mitotic index measured with a previously described technique by Wright et $a l^{(26)}$. In order to verify our protocol we performed a pilot study comparing both methods, which yielded statistically indifferent results (data not shown). The mitosis:apoptosis ratio was determined by dividing the $\mathrm{V}_{\mathrm{vprol}}$ by the $\mathrm{V}_{\text {vapop}}$.

The immunoreactive intensity of the ECMP-stained slices was evaluated using an image analysis program AnalySIS
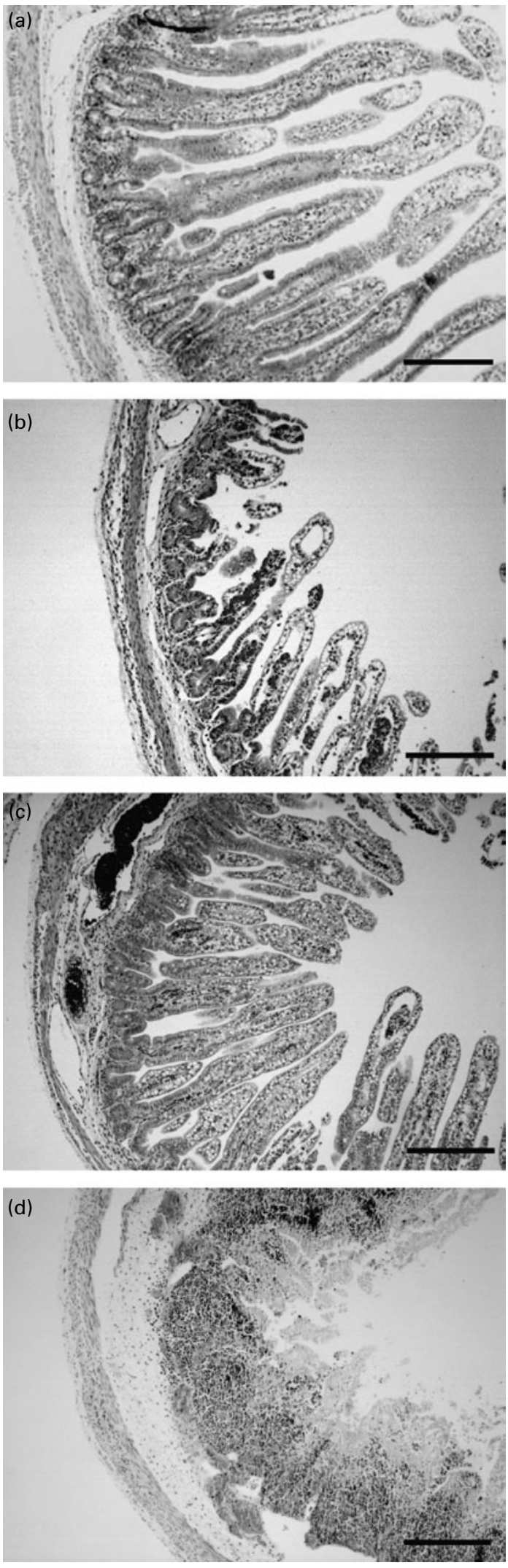

Fig. 1. Haematoxylin and eosin-stained sections of the preterm small intestine, illustrating: (a) no lesions; (b) mild dilated submucosal vessels; (c) severely dilated submucosal vessels; (d) regional loss of villi, severely dilated submucosal vessels and blood congestion. Scale bar $=100 \mu \mathrm{m}$. 
Table 1. Immunohistochemistry

\begin{tabular}{|c|c|c|c|c|}
\hline Target & Pretreatment & $\begin{array}{l}\text { Primary antibody, dilution, } \\
\text { time, temperature }\end{array}$ & $\begin{array}{l}\text { Secondary antibody, dilution, } \\
\text { time, temperature }\end{array}$ & $\begin{array}{l}\text { Peroxidase system, dilution, } \\
\text { time, temperature }\end{array}$ \\
\hline Laminin-1 & $\begin{array}{l}\text { Trypsine (MP Biomedical, } \\
\text { Montréal, Canada), } \\
\mathrm{pH} 7 \cdot 8,20 \text { min, } 37^{\circ} \mathrm{C}\end{array}$ & $\begin{array}{l}\text { Anti-laminin (Sigma, } \\
\text { Bornem, Belgium), } \\
1 / 25,90 \mathrm{~min}, 37^{\circ} \mathrm{C}\end{array}$ & $\begin{array}{l}\text { Biotinylated swine anti-rabbit } \\
\text { (DakoCytomation, Glostrup, } \\
\text { Denmark), 1/200, } 30 \text { min, RT }\end{array}$ & $\begin{array}{l}\text { Streptavidin horseradish } \\
\text { peroxidase (DakoCytomation), } \\
\text { 1/200, } 30 \mathrm{~min}, \mathrm{RT}\end{array}$ \\
\hline Fibronectin & $\begin{array}{l}\text { EDTA, pH } 8,30 \mathrm{~min} \\
92-97^{\circ} \mathrm{C}, 30 \mathrm{~min} \mathrm{CD}\end{array}$ & $\begin{array}{l}\text { Anti-fibronectin (Sigma), } \\
\text { 1/800, ON, RT }\end{array}$ & $\begin{array}{l}\text { Anti-rabbit Envision }{ }^{\circledR} \\
\quad \text { (DakoCytomation), } 30 \mathrm{~min}, \mathrm{RT}\end{array}$ & \\
\hline Collagen IV & $\begin{array}{l}\text { Target retrieval solution } \\
\text { (DakoCytomation), } \\
\text { pH 6, } 10 \text { min, pressure } \\
\text { cooker, } 20 \text { min CD }\end{array}$ & $\begin{array}{l}\text { Anti-human collagen IV } \\
\text { (DakoCytomation), } \\
1 / 25,90 \mathrm{~min}, 37^{\circ} \mathrm{C}\end{array}$ & $\begin{array}{l}\text { Anti-mouse Envision }^{\circledR} \\
\quad \text { (DakoCytomation), } 30 \mathrm{~min}, \mathrm{RT}\end{array}$ & \\
\hline PCNA & $\begin{array}{l}\text { Target retrieval solution } \\
\text { (DakoCytomation), } \\
\text { pH 6, } 10 \text { min, microwave, } \\
10 \text { min CD }\end{array}$ & $\begin{array}{l}\text { PCNA (Sigma), 1/500, } \\
\quad 45 \text { min, RT }\end{array}$ & $\begin{array}{l}\text { Biotinylated goat anti-mouse } \\
\text { (DakoCytomation), 1/200, } \\
30 \text { min, RT }\end{array}$ & $\begin{array}{l}\text { Streptavidin horseradish } \\
\text { peroxidase (DakoCytomation), } \\
\text { 1/200, } 30 \mathrm{~min}, \mathrm{RT}\end{array}$ \\
\hline $\begin{array}{l}\text { Cleaved } \\
\text { caspase-3 protein }\end{array}$ & $\begin{array}{l}\text { Target retrieval solution } \\
\text { (DakoCytomation), } \\
\text { pH 6, 20 min, microwave, } \\
20 \text { min CD }\end{array}$ & $\begin{array}{l}\text { Cleaved caspase } 3 \\
\quad(\text { Sigma }), 1 / 250 \\
120 \mathrm{~min}, 37^{\circ} \mathrm{C}\end{array}$ & $\begin{array}{l}\text { Anti-rabbit Envision }{ }^{\circledR} \\
\quad \text { (DakoCytomation), } 60 \mathrm{~min}, \mathrm{RT}\end{array}$ & \\
\hline EGFR & $\begin{array}{l}\text { Biotinin blocking } \\
\text { (DakoCytomation) }\end{array}$ & $\begin{array}{l}\text { Anti-EGFR (Sigma), } \\
1 / 70, \text { ON }, 4^{\circ} \mathrm{C}\end{array}$ & $\begin{array}{l}\text { Biotinylated goat anti-mouse } \\
\text { (DakoCytomation), 1/400, } \\
30 \mathrm{~min}, \mathrm{RT}\end{array}$ & $\begin{array}{l}\text { Streptavidin horseradish } \\
\text { peroxidase (DakoCytomation), } \\
\text { 1/200, } 30 \mathrm{~min}, \mathrm{RT}\end{array}$ \\
\hline
\end{tabular}

$\mathrm{RT}$, room temperature; CD, crypt depth; ON, overnight; PCNA, proliferating cell nuclear antigen; EGFR, epidermal growth factor receptor.

Pro 5.0 (Olympus). The images were converted into greyscale (black: grey-value $=0$ to white: grey-value $=254$ ). The average of the grey-value of the different parts of the intestinal wall (crypt region and proximal part, middle part and distal part of the villus) was determined. The difference between the grey-value of different parts measured on the same section (for example, grey-value of the distal part of the villus minus grey-value of the proximal part of the villus) was taken and statistically these values were compared between the various experimental groups in order to detect staining differences along the crypt-villus axis. Of each tissue block, approximately sixty crypt-villus axes were scored in a total of thirty sections taken systematically at random.

\section{Statistical evaluation}

The volume densities were arcsine-transformed to turn percentages into an infinite data range. Since no interaction was detected between region or feeding group, the morphological data were analysed by repeated-measures ANOVA (region as in-between factor) to detect significant effects of feeding type. The possibility for regional differences to exist was explored via ANOVA analyses. Post hoc comparisons were performed using a least significant difference (LSD) test.

The grey-value difference of the ECMP did not meet the requirement for using parametric statistical methods and therefore Kruskal-Wallis tests were performed.

Pearson correlation tests were used to detect correlations between the different parameters.

All analyses were carried out using SPSS 16.0 (SPSS, Inc., Chicago, IL, USA).

Unless stated otherwise, the values given are the means of the three intestinal regions. Results are expressed as mean values with their standard errors. $P<0.05$ was considered significant.

\section{Results}

Villus height, crypt depth, crypt fission and lesions

Feeding parenteral nutrition for $3 \mathrm{~d}$ was not associated with significant changes in villus height and crypt depth relative to the UFD piglets $(P>0 \cdot 05)$. In contrast, $2 \mathrm{~d}$ of enteral nutrition with sow's colostrum after $3 \mathrm{~d}$ of TPN induced a significant increase in villus height in the small intestine compared with TPN and formula-fed pigs (values irrespective of intestinal region: TPN, 519 (SEM 35) $\mu \mathrm{m}$; COL, 624 (SEM 29) $\mu \mathrm{m}$; FOR, 521 (SEM 32) $\mu \mathrm{m})(P=0 \cdot 02)$. Irrespective of feeding regimen, villus height was higher in the middle small intestine (549 (SEM 21) $\mu \mathrm{m})$ compared with in the proximal small intestine (483 (SEM 27) $\mu \mathrm{m}$ ) $(P=0 \cdot 04)$ (Table 2).

Crypt depth in enterally fed pigs was higher relative to in the TPN-fed group (values irrespective of intestinal region: TPN, 52 (SEM 3) $\mu \mathrm{m}$; COL, 63 (SEM 3) $\mu \mathrm{m}$; FOR, 65 (SEM 3) $\mu \mathrm{m})(P=0.03)$. Irrespective of feeding regimen, crypt depth was higher in proximal (62 (SEM 3) $\mu \mathrm{m})$ compared with in middle (54 (SEM 2) $\mu \mathrm{m})$ and distal small intestine (52 (SEM 2) $\mu \mathrm{m})(P=0 \cdot 03)$ (Table 2).

Crypt fission (Fig. 2) apparently did not differ between the various groups $(P=0.06)$. Irrespective of feeding regimen, crypt fission was lower in proximal (13.6 (SEM 2.5) \%), compared with in middle (19.9 (SEM 8.0) \%) and distal small intestine (21.9 (SEM 6.7) \%) $(P=0.02)$ (Table 2).

After $3 \mathrm{~d}$ of TPN no significant increase in lesion score was seen compared with the UFD piglets $(P>0 \cdot 05)$. COL piglets $(0.21$ (SEM 0.08)) had a lower lesion score than FOR piglets $(1.04$ (SEM 0.18)) $(P=0.02)$. The microscopic lesion score was positively correlated with the $\mathrm{V}_{\mathrm{vprol}}$ $(P=0.02)$, the $\mathrm{V}_{\text {vapop }}(P=0 \cdot 03)$, and in consequence with the mitosis:apoptosis ratio $(P=0.03)$. Examples of tissues having the characteristics of lesion scores $0-4$ are shown in Fig. 3. 
Table 2. Villus height $(\mu \mathrm{m})$, crypt depth $(\mu \mathrm{m})$, crypt fission (\%), volume density of epithelial proliferating cells $\left(V_{\text {vprol }}\right)(\%)$ and apoptotic cells $\left(\mathrm{V}_{\text {vapop }}\right)(\%)$ according to small-intestinal region and feeding group (Mean values with their standard errors)

\begin{tabular}{|c|c|c|c|c|c|c|}
\hline & \multicolumn{2}{|c|}{ Proximal } & \multicolumn{2}{|c|}{ Middle } & \multicolumn{2}{|c|}{ Distal } \\
\hline & Mean & SEM & Mean & SEM & Mean & SEM \\
\hline \multicolumn{7}{|c|}{ Villus length $(\mu \mathrm{m})$} \\
\hline UFD & 359 & 63 & 414 & 56 & 484 & 63 \\
\hline TPN & 514 & 51 & 536 & 51 & 506 & 51 \\
\hline FOR & 559 & 40 & 564 & 40 & 527 & 40 \\
\hline $\mathrm{COL}$ & 636 & 44 & 674 & 44 & 541 & 51 \\
\hline \multicolumn{7}{|c|}{ Crypt depth ( $\mu \mathrm{m})$} \\
\hline UFD & $45 \cdot 7$ & $5 \cdot 7$ & 41.5 & $5 \cdot 1$ & $52 \cdot 3$ & $5 \cdot 8$ \\
\hline TPN & 58.5 & 4.7 & $49 \cdot 0$ & 4.7 & $48 \cdot 1$ & 4.7 \\
\hline FOR & 77.4 & $3 \cdot 6$ & 59.7 & $3 \cdot 6$ & $58 \cdot 1$ & 3.4 \\
\hline $\mathrm{COL}$ & $70 \cdot 3$ & $4 \cdot 1$ & $62 \cdot 4$ & $4 \cdot 1$ & $58 \cdot 2$ & $4 \cdot 7$ \\
\hline \multicolumn{7}{|c|}{ Crypt fission (\%) } \\
\hline UFD & $14 \cdot 17$ & 1.01 & $16 \cdot 28$ & 1.93 & 12.40 & $1 \cdot 15$ \\
\hline TPN & $13 \cdot 10$ & 0.67 & $14 \cdot 23$ & 1.93 & $16 \cdot 90$ & 2.42 \\
\hline FOR & $13 \cdot 33$ & 4.42 & 17.43 & 1.72 & 18.94 & 0.82 \\
\hline $\mathrm{COL}$ & $13 \cdot 64$ & $1 \cdot 13$ & $19 \cdot 38$ & $3 \cdot 17$ & 23.46 & $2 \cdot 78$ \\
\hline \multicolumn{7}{|c|}{$\mathrm{V}_{\text {vprol }}(\%)$} \\
\hline UFD & $15 \cdot 66$ & 0.34 & $10 \cdot 81$ & 0.05 & $10 \cdot 75$ & 0.04 \\
\hline TPN & $12 \cdot 79$ & 0.75 & $7 \cdot 33$ & 0.36 & 9.05 & 0.12 \\
\hline FOR & $10 \cdot 81$ & 0.08 & 9.64 & 0.22 & $8 \cdot 76$ & 0.74 \\
\hline $\mathrm{COL}$ & $12 \cdot 63$ & 0.32 & $10 \cdot 28$ & 0.50 & $11 \cdot 26$ & 0.79 \\
\hline \multicolumn{7}{|c|}{$\mathrm{V}_{\text {vapop }}(\%)$} \\
\hline UFD & 0.756 & 0.007 & 1.299 & 0.137 & 0.839 & 0.120 \\
\hline TPN & 0.622 & 0.071 & 0.964 & 0.008 & 0.643 & 0.061 \\
\hline FOR & 0.693 & 0.034 & 1.096 & 0.135 & 0.522 & 0.007 \\
\hline $\mathrm{COL}$ & 0.715 & 0.048 & 1.061 & 0.088 & 0.653 & 0.071 \\
\hline
\end{tabular}

UFD, no nutrition; TPN, total parenteral nutrition; FOR, oral nutrition with an infant milk formula, COL, oral nutrition with sow's colostrum.

\section{Volume density of proliferative and apoptotic cells}

Proliferating cell nuclear antigen immunoreactivity was almost entirely confined to the nucleus. The cells in mitotic division were restricted to the crypt compartment in all feeding groups except for occasional mitoses at the transition between crypt and intervillus floor in COL piglets (Fig. 3(a)). In accordance with this observation, the $\mathrm{V}_{\mathrm{vprol}}$ - irrespective of intestinal region - tended to be higher in the intestine of COL piglets (14.1 (SEM 1.2) \%) compared with TPN (10.4 (SEM 1.2) \%) and FOR piglets (10.1 (SEM 0.7) \%) $(P>0.05)$ (Table 2). Irrespective of feeding regimen, $\mathrm{V}_{\mathrm{vprol}}$ was higher in the proximal $(12.8$ (SEM 0.8$) \%)$ than in the middle intestine $\left(10.6\right.$ (SEM 1.0) \%) $(P=0.04)$. A positive correlation of $\mathrm{V}_{\mathrm{vprol}}$ was seen between crypt depth $(P=0.04)$, microscopic lesion score $(P=0.02), \mathrm{V}_{\text {vapop }}(P=0.01)$ and mitosis:apoptosis ratio $(P<0.01)$. In contrast, a negative correlation was seen between $\mathrm{V}_{\mathrm{vprol}}$ and villus length $(P<0 \cdot 01)$.

Cleaved capsase-3, a specific marker for apoptotic cells, was used to detect and localise apoptotic changes in the small intestine of the piglets. Immunoreactivity was found mainly in the nuclei of apoptotic cells. Apoptotic epithelial cells were present over the entire length of the villi but concentrated at the top of the villi (Fig. 3(b)). Few scattered apoptotic cells were seen in the crypts. Individual cells or cell packets on the top of the villi were shed or collapsed a few micrometres above the villi surfaces. Apoptotic cells could also be detected in the lamina propria (these cells together

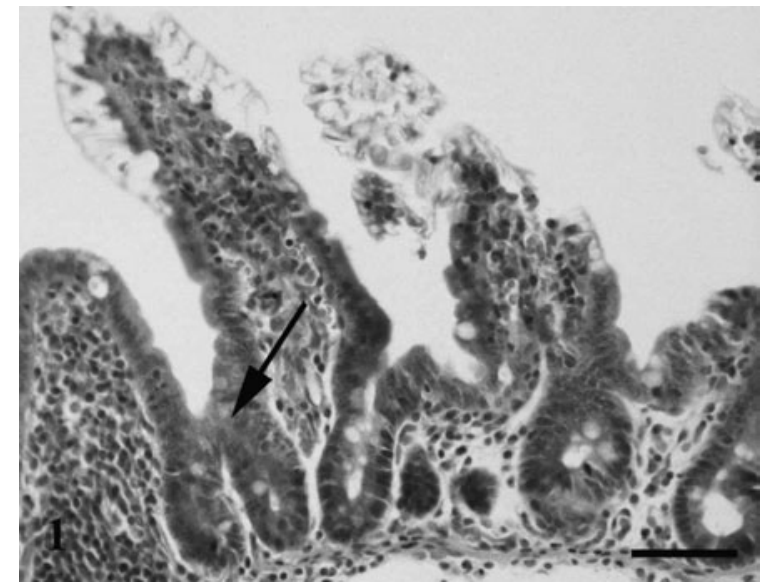

Fig. 2. Crypt fission $(\downarrow)$ in the distal small intestine of a piglet fed orally with sow's colostrum. Scale bar $=50 \mu \mathrm{m}$.

with those shed into the lumen were not taken into account in the measurements). In contrast to $\mathrm{V}_{\mathrm{vprol}}$ no significant differences were seen in $\mathrm{V}_{\text {vapop }}$ between the different feeding groups $(P>0.05)$ (Table 2$)$. Irrespective of feeding group, statistical analyses revealed a higher $\mathrm{V}_{\text {vapop }}$ in the middle intestine $(1 \cdot 1 \quad$ SEM $0 \cdot 1) \%)$ compared with the cranial
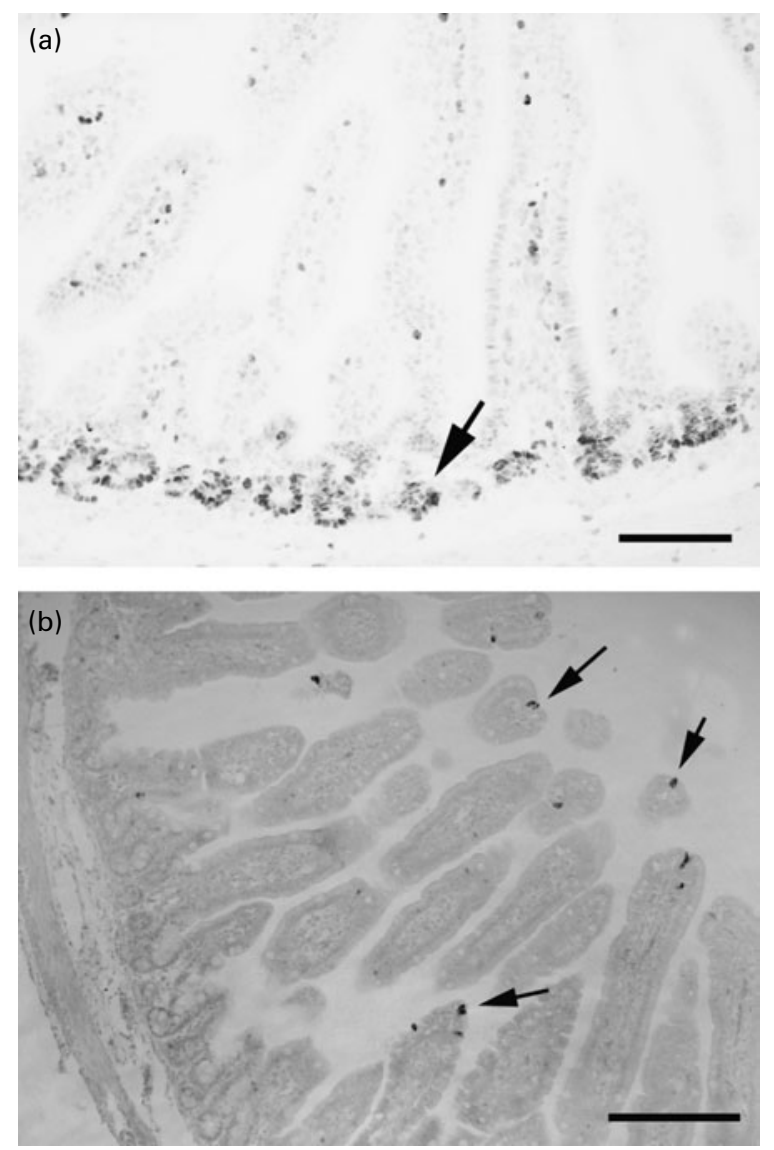

Fig. 3. Proliferating cell nuclear antigen immunoreactivity (a) in epithelial cells in the middle small intestine in an unfed piglet. These epithelial cells were confined to the crypts and the intervillus floor $(\downarrow)$. Scale bar $=100 \mu \mathrm{m}$. Active caspase-3 immunoreactivity (b) in the proximal small intestine of the formula-fed piglet is present at the level of the villus $(\downarrow)$. Scale bar $=200 \mu \mathrm{m}$. 
(0.75 (SEM 0.07) \%) and distal small intestine (0.75 (SEM 0.08) $\%)(P=0.01)$. The $\mathrm{V}_{\text {vapop }}$ was positively correlated with the microscopic lesion score $(P=0.03)$ and $\mathrm{V}_{\mathrm{vprol}}(P=0.01)$.

The $\mathrm{V}_{\text {vprol }}: \mathrm{V}_{\text {vapop }}$ ratio did not significantly differ between the feeding groups. However, the mitosis:apoptosis ratio was significantly lower in the middle (9.3 (SEM 1.4) \%) compared with the proximal (23.4 (SEM 1.4) \%) and distal (24.8 (SEM 1.3) \%) small intestine $(P<0.01)$ irrespective of feeding group.

\section{The extracellular matrix proteins}

Collagen IV immunoreactivity was uniformly stained along the crypt-villus axis (Fig. 4(a)). In some small-intestinal segments of the TPN-fed piglets collagen IV immunoreactivity was less intense. Laminin-1 immunoreactivity (Fig. 4(b)) and fibronectin immunoreactivity (Fig. 4(c)) were homogeneously distributed in the crypt and villus basement membrane. In some small-intestinal segments laminin-1 immunoreactivity and fibronectin immunoreactivity revealed a gradient in their staining intensity. The staining intensity of laminin-1 immunoreactivity expressed a decrease from the villus to the crypt whereas the staining intensity of fibronectin immunoreactivity increased from the apex of the villus towards the crypts in some of the sections. However, these distribution patterns could not be assigned to a specific feeding pattern or histopathological scoring $(P>0 \cdot 05)$.

\section{Discussion}

Numerous studies have shown that TPN induces mucosal atrophy in term neonates, which has been attributed to the absence of trophic signals from the lack of luminal nutrients ${ }^{(27-29)}$ and a drop in intestinal blood flow ${ }^{(5)}$. Nevertheless, TPN is often instituted in preterm neonates because the immature intestine may not tolerate enteral feeding ${ }^{(2)}$. Fortunately, the TPN-fed preterm intestine does not appear to suffer from the same degree of mucosal atrophy as the term intestine although TPN remains inferior in stimulating gut growth and maturation compared with enteral nutrition ${ }^{(1,3,4)}$. In the present study using the preterm piglet, the lack of any effect of $3 \mathrm{~d}$ of TPN on villus height and crypt depth confirms these previous observations. In addition, we found that the underlying mechanisms such as the distribution of ECMP, apoptosis, mitosis, and crypt fission remained unchanged in the TPN fed.

Quantitative image analysis revealed that type IV collagen, fibronectin and laminin-1 were uniformly distributed along the crypt-villus axis. This contrasts with the results of Groos et al., who describe specific changes in the distribution pattern of laminin-1, fibronectin and collagen IV in human adult intestine after parenteral feeding ${ }^{(30)}$. Taken together, the present results suggest that preterm piglets do not exhibit TPNinduced mucosal atrophy after $3 \mathrm{~d}$ of TPN, partly due to indifferent proliferation and apoptosis indices. The latter could be the result of the distribution pattern of the ECMP that resembles mainly that of a fetal (immature) intestine ${ }^{(31,32)}$.

In term piglets, $1 \mathrm{~d}$ of suckling rapidly increases the enterocyte proliferation rate and decreases the apoptotic rate ${ }^{(8,33)}$ In contrast, $2 \mathrm{~d}$ enteral feeding with colostrum induced an increase of the $V_{\text {vprol }}$ but no change in the $V_{\text {vapop }}$ in the preterm piglet's intestine. Significant apoptosis-inducing
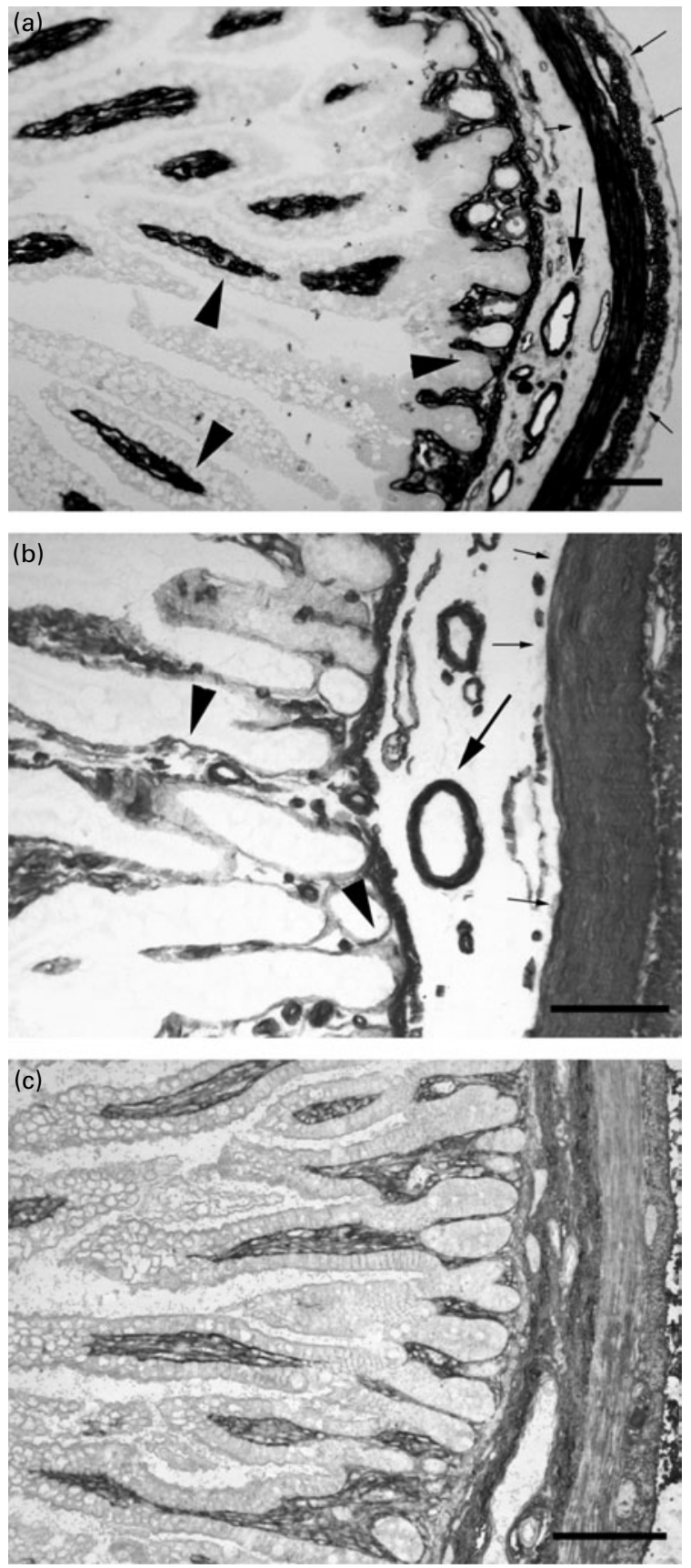

Fig. 4. Presence of collagen IV immunoreactivity in the middle part (a), laminin-1 immunoreactivity in the distal part (b) and fibronectin immunoreactivity in the middle part (c) of the small intestine of a formula-fed piglet. Collagen IV immunoreactivity and laminin immunoreactivity were uniformly present along the crypt-villus axis $(\boldsymbol{\nabla})$, in the wall of the blood vessels $(\downarrow)$ and in the muscle layer $(\rightarrow, \leftarrow)$. Fibronectin immunoreactivity was present in the connective tissue of the lamina propria, submucosal and muscle layer. Scale bar $=100 \mu \mathrm{m}$

mechanisms during normal physiological development of porcine gut epithelium are the local deprivation of growth factors such as epidermal growth factor ${ }^{(15,34)}$, insulin-like growth factor $^{(35)}$ and leptin ${ }^{(8)}$ and the establishment of an intestinal microbiota ${ }^{(36)}$. This is supported by the observation that a higher apoptosis rate was seen in formula-fed neonatal piglets $^{(12)}$ which received no milk-borne growth factors and 
are known to harbour a different microbiota compared with sow-reared piglets ${ }^{(1,37)}$. However, a difference in apoptotic index between formula-fed and piglets fed colostral milk was not observed in our experimental setting using preterm piglets. Perhaps, the preterm small intestine is lacking certain receptors for food bioactive components or microbiota? It has already been reported that short-term epidermal growth factor treatment was not able to preserve villus architecture in the preterm pig small intestine ${ }^{(1)}$, probably due to a lack of epidermal growth factor receptors ${ }^{(38)}$.

An additional explanation for the unaltered $V_{\text {vapop }}$ in both enterally fed groups could be the unaffected distribution pattern of the ECMP, especially laminin-1. In the mature small intestine, laminin-1 is highly concentrated in the villus region $^{(18,19,30)}$. This is in agreement with the task assigned to this glycoprotein, namely to promote intestinal epithelial proliferation and differentiation ${ }^{(17-20)}$. To facilitate this, the expression of laminin-1 increases from the crypt to the villus region $^{(18,19,30)}$. This gradient was absent in the preterm piglets. Thus, cell differentiation and ultimate shedding from the villus tips is equally possible along the villus axis. Similar to laminin-1, the distribution pattern of fibronectin and collagen IV was unaffected by diet and feeding route. Fibronectin is normally expressed concomitantly with cell migration ${ }^{(18,39)}$. In the preterm piglets, fibronectin immunoreactivity evenly delineates the epithelial basement membrane along the crypt-villus axis. The latter is comparable with the expression pattern in the fetal intestine of piglets ${ }^{(39)}$. The homogeneous distribution of subepithelial collagen corresponds to its role as a biomechanical scaffold ${ }^{(18)}$, and accords to its presence in the human ${ }^{(34)}$ and rat $^{(32)}$ developing gut. The absence of any gradient in ECMP distribution along the villi and equal distribution patterns between the feeding groups can - at least in part - be held responsible for a slow cell migration along the crypt-villus axis.

In consequence of the higher $\mathrm{V}_{\mathrm{vprol}}$ and indifferent $\mathrm{V}_{\text {vapop}}$, the villus length in the colostrum-fed piglets was approximately $20 \%$ higher compared with the length observed in TPN- and formula-fed piglets. This growth could be mediated via crypt hyperplasia, which is characterised by deeper crypts, and/or by crypt fission. However, no significant changes in crypt depth and fission were seen between the enterally fed groups. These morphometric findings contrast with observations in the human neonate. Compared with breast-fed infants, formula-fed infants generally show longer villi, deeper crypts, fewer bifid crypts and a higher mitotic count ${ }^{(40)}$. Thus, our observations of an invariable crypt depth and invariable presence of bifid crypts are somewhat surprising. Burrin et al. clearly showed in term piglets that the trophic response towards sow's colostrum is reflected by deeper crypts ${ }^{(41)}$. Others have related the presence of a high amount of growth factors in colostral milk to a stimulation of crypt fission and as such a stimulation of epithelial growth $^{(42)}$, whereas new data suggest crypt fission to occur independent from food bioactive components ${ }^{(43)}$. In addition, the unchanged crypt depth and fission, relatively small increase in $\mathrm{V}_{\text {vprol }}$ and relatively small increase in villus length observed in the present study contrast with larger differences that are observed in term piglets ${ }^{(7,8,33,35,41,44)}$. This suggests that the trophic response towards enteral feeding is lacking or much slower in the preterm small intestine.
The observation of an increase in villus length could indicate, however, that changes in crypt depth, fission and $\mathrm{V}_{\mathrm{vprol}}$ have occurred in an early phase of enteral feeding. Zhang et al. revealed that $6 \mathrm{~h}$ of suckling is sufficient to cause significant changes in intestinal structure and function ${ }^{(45)}$.

Irrespective of the feeding regimen, typical regional differences could be seen along the small intestine. The mitosis:apoptosis ratio was significantly higher in the proximal intestine, resulting in deeper crypts, fewer bifid crypts and smaller villi. These findings reflect a more efficient proximal absorption of enteral nutrients, resulting in diminished exposure in the distal intestine. In addition, in the proximal small intestine of the preterm piglet, the process of crypt hyperplasia seems to be more important than crypt fission, whereas in the distal small intestine this seems to be reversed.

Consistent with earlier results ${ }^{(1,3,4)}$, TPN fluid infused parenterally is well tolerated by preterm piglets, resulting in an unchanged microscopic lesion score compared with the unfed piglets. Compared with colostrum-fed piglets, formula-fed piglets had less preserved villus architecture and in some cases even complete destruction of the villi (resembling lesions that are associated with those of necrotising enterocolitis). Studies with pigs born at term showed that formula was associated with only minor gastrointestinal dysfunctions at a subclinical level. In addition, feeding formula to term piglets mimicked many of the short-term postnatal trophic effects observed in colostrum-fed piglets ${ }^{(1,3,4)}$. Thus, preterm delivery compromises the intestinal responses to feeding, perhaps resulting in the development of intestinal lesions. During the development of these mucosal lesions, apoptosis may account for the initial loss of cells in the apical villi ${ }^{(46)}$. Moreover, in order to preserve villus architecture, a higher $\mathrm{V}_{\mathrm{vprol}}$ is needed, resulting in a positive correlation between microscopic lesion score, $\mathrm{V}_{\text {vprol }}$ and $\mathrm{V}_{\text {vapop}}$.

In conclusion, the present morphometric study demonstrates that quantitative differences exist with respect to morphological (villus height and crypt depth) and functional $\left(\mathrm{V}_{\mathrm{vprol}}\right.$ and $\mathrm{V}_{\text {vapop}}$, mitosis:apoptosis ratio, crypt fission) parameters between differently fed preterm piglets. TPN feeding for a short period does not affect mitosis and apoptosis rates of enterocytes that perhaps could be related to the preserved fetal distribution pattern of ECMP. Formula feeding to preterm piglets was associated with a shorter villi, a lower $\mathrm{V}_{\mathrm{vprol}}$ and a higher lesion score, relative to colostrum feeding. Nevertheless, deepening of the crypts after formula feeding suggests a trophic, repair response of the intestinal epithelium. In order to understand these morphometric changes in the preterm small intestine, short-term changes should be investigated.

\section{Acknowledgements}

The present study was supported by a research grant of the Fund for Scientific Research-Flanders Belgium (1.5.096.04N) granted to C. V. G. In addition, the authors gratefully acknowledge the support of the Industrial Research Council of the University of Antwerp (2992) granted to C. V. G.

M. O. gathered the morphometrical data and wrote the manuscript with significant contributions by M. D. V., T. T., P. S. and C. V. G.; M. D. V. gathered and interpreted the 
data regarding crypt fission; E. V. H. contributed to the statistical analyses; L. V. B. and A. W. conducted the histopathological scoring; T. T., P. S. and C. V. G. - together with M. O. - designed and conducted the animal experimental protocol. In addition, the authors gratefully acknowledge the excellent technical assistance of Katty Huybrechts and Gunther Vrolix.

The authors state that there is no conflict of interest.

\section{References}

1. Sangild PT (2006) Gut responses to enteral nutrition in preterm infants and animals. Exp Biol Med 231, 1695-1711.

2. Hay WW (2008) Strategies for feeding the preterm infant. Neonatology 94, 245-254.

3. Sangild PT, Petersen YM, Schmidt M, et al. (2002) Preterm birth affects the intestinal response to parenteral and enteral nutrition in newborn pigs. $J$ Nutr 132, 2673-2681.

4. Sangild PT, Siggers RH, Schmidt M, et al. (2006) Diet- and colonization-dependent intestinal dysfunction predisposes to necrotizing enterocolitis in preterm pigs. Gastroenterology 130, 1776-1792.

5. Niinikoski H, Stoll B, Guan X, et al. (2004) Onset of small intestinal atrophy is associated with reduced intestinal blood flow in TPN-fed neonatal piglets. J Nutr 134, 1467-1474.

6. Xu RJ (1996) Development of the newborn gastrointestinal tract and its relation to colostrum/milk intake: a review. Reprod Fertil Dev 8, 35-48.

7. Jensen AR, Elnif J, Burrin DG, et al. (2001) Development of intestinal immunoglobulin absorption and enzyme activities in neonatal pigs is diet dependent. $J$ Nutr 131, 3259-3265.

8. Wolinski J, Biernat M, Guilloteau P, et al. (2003) Exogenous leptin controls the development of the small intestine in neonatal piglets. $J$ Endocrinol 177, 215-222.

9. Berlanga-Acosta J, Playford RJ, Mandir N, et al. (2001) Gastrointestinal cell proliferation and crypt fission are separate but complementary means of increasing tissue mass following infusion of epidermal growth factor in rats. Gut 48, 803-807.

10. Cummins AG, Jones BJ \& Thompson FM (2006) Postnatal epithelial growth of the small intestine in the rat occurs by both crypt fission and crypt hyperplasia. Dig Dis Sci 51, 718-723.

11. Domeneghini C, Di Giancamillo A, Bosi G, et al. (2006) Can nutraceuticals affect the structure of intestinal mucosa? Qualitative and quantitative microanatomy in L-glutamine dietsupplemented weaning piglets. Vet Res Comm 30, 331-342.

12. Godlewski MM, Slupecka M, Wolinski J, et al. (2005) Into the unknown - the death pathways in the neonatal gut epithelium. J Physiol Pharmacol 56, 7-24.

13. Raab S, Leiser R, Kemmer H, et al. (1998) Effects of energy and purines in the diet on proliferation, differentiation, and apoptosis in the small intestine of the pig. Metabolism 47, $1105-1111$.

14. Cummins AG \& Thompson FM (2002) Effect of breast milk and weaning on epithelial growth of the small intestine in humans. Gut 51, 748-754.

15. Park HS, Goodlad RA, Ahnen DJ, et al. (1997) Effects of epidermal growth factor and dimethylhydrazine on crypt size, cell proliferation, and crypt fission in the rat colon. Cell proliferation and crypt fission are controlled independently. Am J Pathol 151, 843-852.

16. Sasaki M, Fitzgerald AJ, Grant G, et al. (2002) Lectins can reverse the distal intestinal atrophy associated with elemental diets in mice. Aliment Pharmacol Ther 16, 633-642.

17. Zhang J, Li W, Sanders MA, et al. (2003) Regulation of the intestinal epithelial response to cyclic strain by extracellular matrix proteins. FASEB J 17, 926-928.
18. Groos S, Hünefeld G \& Luciano L (2001) Epithelial cell turnover - extracellular matrix relationship in the small intestine of human adults. Ital J Anat Embryol 106, 353-361.

19. Teller IC, Auclair J, Herring E, et al. (2007) Laminins in the developing and adult human small intestine: relation with the functional absorptive unit. Dev Dyn 236, 1980-1990.

20. Sato T, Vries RG, Snippert HJ, et al. (2009) Single Lgr5 stem cells build crypt-villus structures in vitro without a mesenchymal niche. Nature 459, 262-265.

21. Hay ED (1981) Extracellular matrix. J Cell Biol 91, 205s-223s.

22. Brenmoehl J, Falk W, Goke M, et al. (2008) Inflammation modulates fibronectin isoform expression in colonic lamina propria fibroblasts (CLPF). Int J Colorectal Dis 23, 947-955.

23. Goke M, Zuk A \& Podolsky DK (1996) Regulation and function of extracellular matrix intestinal epithelial restitution in vitro. Am J Physiol 271, G729-G740.

24. Dvorak B, Halpern MD, Holubec H, et al. (2002) Epidermal growth factor reduces the development of necrotizing enterocolitis in a neonatal rat model. Am $J$ Physiol 282, G156-G164.

25. Gundersen HJG \& Jensen EB (1987) The efficiency of systematic sampling in stereology and its prediction. J Microsc 147, 229-263.

26. Wright N, Watson A, Morley A, et al. (1973) Cell kinetics in flat (avillous) mucosa of the human small intestine. Gut 14, 701-710.

27. Dudley MA, Wykes LJ, Dudley AW, et al. (1998) Parenteral nutrition selectively decreases protein synthesis in the small intestine. Am J Physiol 274, G131-G137.

28. Goldstein RM, Hebiguchi T, Luk GD, et al. (1985) The effects of total parenteral nutrition on gastrointestinal growth and development. J Pediatr Surg 20, 785-791.

29. Shulman RJ (1988) Effect of different total parenteral nutrition fuel mixes on small intestinal growth and differentiation in the infant miniature pig. Gastroenterology 95, 85-92.

30. Groos S, Reale E, Hünefeld G, et al. (2003) Changes in epithelial cells turnover and extracellular matrix in human small intestine after TPN. J Surg Res 109, 74-85.

31. Beaulieu JF, Vachon PH \& Chartrand S (1991) Immunolocalization of extracellular matrix components during organogenesis in the human small intestine. Anat Embryol (Berl) 183, $363-369$.

32. Simon-Assmann P, Kedinger M, De Arcangelis A, et al. (1995) Extracellular matrix components in intestinal development. Experientia 51, 883-900.

33. Zabielski R, Godlewski MM \& Guilloteau P (2008) Control of development of gastrointestinal system in neonates. $J$ Physiol Pharmacol 59, Suppl. 1, 35-54.

34. Clark JA, Lane RH, Maclennan NK, et al. (2005) Epidermal growth factor reduces intestinal apoptosis in an experimental model of necrotizing enterocolitis. Am J Physiol Gastrointest Liver Physiol 288, G755-G762.

35. Xu RJ, Mellor DJ, Birtles MJ, et al. (1994) Effects of oral IGF-I or IGF-II on digestive organ growth in newborn piglets. Biol Neonate 66, 280-287.

36. Willing BP \& Van Kessel AG (2007) Enterocyte proliferation and apoptosis in the caudal small intestine is influenced by the composition of colonizing commensal bacteria in the neonatal gnotobiotic pig. J Anim Sci 85, 3256-3266.

37. Van Haver ER, Oste M, Siggers JLA, et al. (2009) Dietdependent mucosal colonisation and interleukin- $1 \beta$ responses in preterm pigs susceptible to nectrotising enterocolitis. J Pediatr Gastroenterol Nutr 49, 90-98.

38. Van Ginneken C, Van Haver E, Oste M, et al. (2007) The presence of EGF- and IGF-1-receptors in the small intestine of fetal, neonatal and weaned piglets. Livest Sci 108, 57-60. 
39. Trebichavsky I \& Nyklicek O (1995) Distribution of fibronectin in foetal tissues. Cell Biol Int 19, 813-819.

40. Thompson FM, Catto-Smith AG, Moore D, et al. (1998) Epithelial growth of the small intestine in human infants. J Pediatr Gastroenterol Nutr 26, 506-512.

41. Burrin DG, Davis TA, Ebner S, et al. (1995) Nutrientindependent and nutrient-dependent factors stimulate protein synthesis in colostrum-fed newborn pigs. Pediatr Res 37, 593-599.

42. Cummins AG \& Thompson FM (2002) Effect of breast milk and weaning on epithelial growth of the small intestine in humans. Gut 51, 748-754.
43. Slupecka M, Wolinski J \& Pierzynowski SG (2010) Crypt fission contributes to postnatal epithelial growth of the small intestine in pigs. Livest Sci (In the Press).

44. Xu RJ, Mellor DJ, Tungthanatanich P, et al. (1992) Growth and morphological changes in the small and large intestine in piglets during the first three days after birth. J Dev Physiol 18, 161-172.

45. Zhang H, Malo C \& Buddington RK (1997) Suckling induces rapid intestinal growth and changes in brush border digestive functions of newborn pigs. J Nutr 127, 418-426.

46. Jilling T, Lu J, Jackson M, et al. (2004) Intestinal epithelial apoptosis initiates gross bowel necrosis in an experimental rat model of neonatal necrotizing enterocolitis. Pediatr Res 55, 622-629. 